\title{
LINEAR FUNCTIONAL OPERATIONS ON FUNCTIONS HAVING DISCONTINUITIES OF THE FIRST KIND*
}

\author{
BY H. S. KALTENBORN
}

The most general form of a linear continuous functional operation on a function $f(x)$ continuous on $(a, b)$ has been expressed by F. Riesz $\dagger$ as a Stieltjes integral of $f(x)$ with respect to a function of bounded variation. By virtue of the extension theorem of Hahn, $\ddagger$ this result can be extended to the case where $f(x)$ has discontinuities of the first kind on $(a, b)$. In establishing this more general case, the modified form of the integral considered by B. Dushnik $\S$ is used instead of the ordinary Stieltjes integral. The expression obtained for $T[f]$ reduces to that given by Riesz when the function $f(x)$ is continuous since in this case the modified integral agrees with the ordinary integral.

The following definitions and properties enter into the statement and proof of the theorem. Let $f$ denote an element of a linear vector space $S$ of norm $\|f\| ;$ then an operation $T$ on $S$ is linear if

$$
T\left[k_{1} f_{1}+k_{2} f_{2}\right]=k_{1} T\left[f_{1}\right]+k_{2} T\left[f_{2}\right],
$$

where the $k$ 's are any constants; and $T$ is bounded if there exists an $M>0$ depending on $T$ such that

$$
|T[f]| \leqq M\|f\| \text { for every } f \text { of } S .
$$

The condition of boundedness (2) is equivalent** to the property of continuity

* Presented to the Society, April 6, 1934.

$\dagger$ Annales de l'École Normale Supérieure, (3), vol. 31 (1914), pp. 9-14. Two new proofs of this theorem have been given recently by T. H. Hildebrandt and I. J. Schoenberg, Annals of Mathematics, vol. 34 (1933), pp. 317319.

$\ddagger$ See S. Banach, Théorie des Opérations Linéaires, 1932, pp. 55, 59.

$\S$ Dissertation, University of Michigan, 1931.

I See T. H. Hildebrandt, Linear functional transformations in general spaces, this Bulletin, vol. 37 (1931), p. 186.

** See S. Banach, loc. cit., p. 54. 


$$
\lim _{n \rightarrow \infty} T\left[f_{n}\right]=T[f], \text { when } \lim _{n \rightarrow \infty}\left\|f_{n}-f\right\|=0 \text {. }
$$

The class $C$ of all continuous functions on $(a, b)$, and the class $D$ of functions having discontinuities of the first kind constitute linear vector spaces whose norms are the maximum and least upper bound, respectively, of $|f(x)|$ on $(a, b)$. The modified form of the integral that will be used is defined by

$$
\int_{a}^{b} f d \alpha=\lim \sum_{i=1}^{n} f\left(\xi_{i}\right)\left[\alpha\left(x_{i}\right)-\alpha\left(x_{i-1}\right)\right],
$$

where $a=x_{0}<x_{1}<x_{2}<\cdots<x_{n}=b$ is any subdivision of $(a, b), \xi_{i}$ is an arbitrary inner point of $\left(x_{i-1}, x_{i}\right)$, and the limit is taken as the subdivision is made successively finer.* The symbol $\int_{a}^{b} f d \alpha$ will be used throughout this paper to denote an integral of this type.

An analog of Riesz's theorem for functions of class $D$ is then contained in the following theorem.

TheOREM. Every linear continuous operation $T$ applied to a function $f(x)$ of $D$ may be expressed in the form

$$
T[f]=\int_{a}^{b} f d \psi+\sum_{i=0}^{\infty}\left[f\left(c_{i}\right)-f\left(c_{i}-0\right)\right] \phi\left(c_{i}\right),
$$

where $c_{0}, c_{1}, c_{2}, \cdots$ denote the points of discontinuity of $f(x)$, $c_{0}=a, \psi(x)$ and $\phi(x)$ depend only on $T$ and are such that $\psi(x)$ is of bounded variation and $\phi(x)$ vanishes except at a denumerable set of points and the sum of the absolute values of $\phi(x)$ at these points is finite.

It will be noted that the expression for $T[f]$ depends on subdivisions of $(a, b)$ into sets of open intervals and single points rather than on subdivisions into closed intervals as is the case when $f(x)$ is continuous and the ordinary integral is used. The first term on the right in (5) corresponds to the open intervals of the subdivision, and the second term arises from the points of discontinuity of $f(x)$. If $f(x)$ is continuous, the second term vanishes, and the expression for $T[f]$ reduces to

* See H. L. Smith, Transactions of this Society, vol. 27 (1925), pp. 491-492. The integral (4) differs from Smith's integral $F S \int f d \alpha$ in that $\xi_{i}$ is restricted to be an inner point of $\left(x_{i-1}, x_{i}\right)$. 
the modified integral $\int_{a}^{b} f d \psi$. Now the ordinary Stieltjes integral exists since $f(x)$ is continuous and $\psi(x)$ is of bounded variation, and since the modified integral equals the ordinary integral when the latter exists, it follows that the form for $T[f]$ in this case is the same as that obtained by Riesz.

To obtain the functions $\phi(x)$ and $\psi(x)$ of (5), introduce the functions $g(t, x)=1$ or 0 according as $t=x$ or $t \neq x$, and $h(t, x)$ $=1$ or 0 according as $a \leqq t \leqq x$ or $x<t \leqq b$, and then place

$$
\phi(x)=T[g(t, x)], \quad \psi(x)=T[h(t, x)] .
$$

The properties of $\phi(x)$ and $\psi(x)$ stated in the theorem follow from these definitions. Thus if $x_{1}, x_{2}, \cdots, x_{n}$ is any finite set of points of $(a, b)$, then from (2) and the definition of $g(t, x)$,

$$
\begin{aligned}
\sum_{i=1}^{n}\left|\phi\left(x_{i}\right)\right| & =\sum_{i=1}^{n} \operatorname{sgn} \phi\left(x_{i}\right) T\left[g\left(t, x_{i}\right)\right] \\
& \leqq M\left\|\sum_{i=1}^{n} \operatorname{sgn} \phi\left(x_{i}\right) g\left(t, x_{i}\right)\right\|=M .
\end{aligned}
$$

Consequently $\phi(x)$ vanishes except at a denumerable set of points, and the sum of the absolute values of $\phi(x)$ at these points is $\leqq M$. The function $\psi(x)$ is of bounded variation, since for any subdivision of $(a, b)$,

$$
\begin{aligned}
\sum_{i=1}^{n}\left|\psi\left(x_{i}\right)-\psi\left(x_{i-1}\right)\right| & =\sum_{i=1}^{n}\left|T\left[h\left(t, x_{i}\right)-h\left(t, x_{i-1}\right)\right]\right| \\
& \leqq M\left\|\sum_{i=1}^{n}\left[h\left(t, x_{i}\right)-h\left(t, x_{i-1}\right)\right]\right\|=M .
\end{aligned}
$$

The given function $f(x)$ may be uniformly approximated on $(a, b)$ by means of a sequence of functions of the type

$$
\begin{aligned}
f_{\sigma}(x)= & \sum_{i=1}^{n} f\left(\xi_{i}\right)\left[h\left(x, x_{i}\right)-h\left(x, x_{i-1}\right)-g\left(x, x_{i}\right)\right] \\
& +\sum_{i=0}^{n} f\left(x_{i}\right) g\left(x, x_{i}\right) \\
= & \sum_{i=1}^{n} f\left(\xi_{i}\right)\left[h\left(x, x_{i}\right)-h\left(x, x_{i-1}\right)\right] \\
& +\sum_{i=0}^{n}\left[f\left(x_{i}\right)-f\left(\xi_{i}\right)\right] g\left(x, x_{i}\right)
\end{aligned}
$$


where $\sigma=\left(a=x_{0}<x_{1}<x_{2}<\cdots<x_{n}=b\right)$ is a finite subdivision of $(a, b), \xi_{i}$ is an arbitrary inner point of $\left(x_{i-1}, x_{i}\right)$, and $f\left(\xi_{0}\right)=0$. For if $\epsilon>0$ denotes an arbitrarily small quantity, then a subdivision $\sigma_{\epsilon}$ of $(a, b)$ can be found* so that the oscillation of $f(x)$ on the interior of any subinterval $\left(x_{i-1}, x_{i}\right),(i=1,2, \cdots$, $n)$, is less than $\epsilon$. Since $f_{\sigma}\left(x_{i}\right)=f\left(x_{i}\right)$, and $f_{\sigma}(x)=f\left(\xi_{i}\right)$ for $x_{i-1}$ $<x<x_{i},(i=1,2, \cdots, n)$, then if $\sigma$ is a finer subdivision than $\sigma_{\epsilon},\left|f(x)-f_{\sigma}(x)\right|<\epsilon$ for any $x$ on $(a, b)$, and hence $\lim f_{\sigma}(x)$ $=f(x)$ uniformly. Therefore, from (3),

$$
\lim T\left[f_{\sigma}(x)\right]=T[f(x)] .
$$

Applying $T$ to (7), and making use of (6), we get

$$
T\left[f_{\sigma}\right]=\sum_{i=1}^{n} f\left(\xi_{i}\right)\left[\psi\left(x_{i}\right)-\psi\left(x_{i-1}\right)\right]+\sum_{i=0}^{n}\left[f\left(x_{i}\right)-f\left(\xi_{i}\right)\right] \phi\left(x_{i}\right) .
$$

The limit of the first sum on the right exists $\dagger$ and is equal to $\int_{a}^{b} f d \psi$. Therefore the limit of the second sum on the right exists, and we have

$$
T[f]=\int_{a}^{b} f d \psi+\lim \sum_{i=0}^{n}\left[f\left(x_{i}\right)-f\left(\xi_{i}\right)\right] \phi\left(x_{i}\right) .
$$

Let $Q$ denote the sum on the right for a subdivision finer than $\sigma_{\epsilon}$. Then $\left|f\left(x_{i}-0\right)-f\left(\xi_{i}\right)\right|<\epsilon,(i=0,1,2, \cdots, n)$, and hence

$$
\begin{aligned}
\mid Q- & \sum_{i=0}^{n}\left[f\left(x_{i}\right)-f\left(x_{i}-0\right)\right] \phi\left(x_{i}\right) \mid \\
& =\left|\sum_{i=0}^{n}\left[f\left(x_{i}-0\right)-f\left(\xi_{i}\right)\right] \phi\left(x_{i}\right)\right| \\
& \leqq \epsilon \sum_{i=0}^{n}\left|\phi\left(x_{i}\right)\right| \leqq \epsilon M,
\end{aligned}
$$

from which

$$
\lim Q=\lim \sum_{i=0}^{n}\left[f\left(x_{i}\right)-f\left(x_{i}-0\right)\right] \phi\left(x_{i}\right)
$$

* H. Hahn. Theorie der Reellen Funktionen, vol. 1, 1921, p. 217.

$\dagger$ Dushnik, loc. cit. 
Since $\left|f\left(c_{i}\right)-f\left(c_{i}-0\right)\right|<\epsilon$ for every $c_{i}$ which is not a point of $\sigma_{\epsilon}$, then there is an integer $m \leqq n$ such that, if $\sum^{\prime}$ denotes summation over the points of $\sigma$ which are not points of discontinuity of $f(x)$,

$$
\begin{aligned}
& \left|\sum_{i=0}^{\infty}\left[f\left(c_{i}\right)-f\left(c_{i}-0\right)\right] \phi\left(c_{i}\right)-\sum_{i=0}^{n}\left[f\left(x_{i}\right)-f\left(x_{i}-0\right)\right] \phi\left(x_{i}\right)\right| \\
& =\mid \sum_{i=m}^{\infty}\left[f\left(c_{i}\right)-f\left(c_{i}-0\right)\right] \phi\left(c_{i}\right)-\sum^{\prime}\left[f\left(x_{i}\right)-f\left(x_{i}-0\right)\left|\phi\left(x_{i}\right)\right|\right. \\
& \leqq \sum_{i=m}^{\infty}\left|f\left(c_{i}\right)-f\left(c_{i}-0\right)\right|\left|\phi\left(c_{i}\right)\right| \\
& \quad+\sum^{\prime}\left|f\left(x_{i}\right)-f\left(x_{i}-0\right)\right|\left|\phi\left(x_{i}\right)\right| \leqq 2 \epsilon M .
\end{aligned}
$$

Therefore,

$$
\lim Q=\sum_{i=0}^{\infty}\left[f\left(c_{i}\right)-f\left(c_{i}-0\right)\right] \phi\left(c_{i}\right) .
$$

Substituting this value of $\lim Q$ in (8), we obtain (5), and thus the theorem is proved.

If the function $\chi(x)=T[k(t, x)]$, where $k(t, x)=0$ or 1 according as $a \leqq t<x$ or $x \leqq t \leqq b$, is used instead of $\psi(x)$, the expression

$$
T[f]=\int_{a}^{b} f d \chi+\sum_{i=0}^{\infty}\left[f\left(c_{i}\right)-f\left(c_{i}+0\right)\right] \phi\left(c_{i}\right)
$$

is obtained instead of (5). To obtain a more general form of $T[f]$, which includes both (5) and (9), multiply (5) by $\lambda$ and (9) by $1-\lambda$, and add. Then

$$
\begin{aligned}
T[f]=\int_{a}^{b} f d \theta & +\sum_{i=0}^{\infty}\left[f\left(c_{i}\right)-\left\{\lambda f\left(c_{i}-0\right)\right.\right. \\
& \left.\left.+(1-\lambda) f\left(c_{i}+0\right)\right\}\right] \phi\left(c_{i}\right),
\end{aligned}
$$

where $\theta(x)=\lambda \psi(x)+(1-\lambda) \chi(x)$. Certain conditions under which $T[f]$ can be expressed directly as a modified integral without any corrective terms being necessary may be seen at once from the equation (10). This is the case if $T$ is such that $\phi(x) \equiv 0$, or if*

* This case is considered by H. Hahn, Monatshefte für Mathematik und Physik, vol. 32 (1922), p. 53. 
$f(x)=\lambda f(x-0)+(1-\lambda) f(x+0)$. In particular, $T[f]$ is expressible just as an integral if $T$ operates on the class of functions $f(x)$ which are continuous on the right (or on the left) throughout $(a, b)$, or for which $f(x)=(1 / 2)(f(x-0)+f(x+0))$ at every point.

If $\phi(x)=(1 / 2)(\psi(x+0)-\psi(x-0))$ at every point, for the operation $T$, then $T[f]$ is expressible directly as a Stieltjes mean integral,

$$
T[f]=(m) \int_{a}^{b} \cdot f d \alpha,
$$

where $\alpha(x)=2 \psi(x)-\psi(x+0)$, and the integral is obtained from (4) by replacing $f\left(\xi_{i}\right)$ by $(1 / 2)\left(f\left(x_{i-1}\right)+f\left(x_{i}\right)\right)$. Comparing (5) and (11) for $f(x)=g(t, x)$ and $f(x)=h(t, x)$, we get as necessary and sufficient conditions for their equivalence,

$$
\begin{aligned}
& \phi(x)=\frac{1}{2}[\alpha(x+0)-\alpha(x-0)], \\
& \psi(x)=\frac{1}{2}[\alpha(x+0)+\alpha(x)] .
\end{aligned}
$$

Since $\alpha(x)$ is continuous except at a denumerable set of points, it follows from the second of equations (12) that $\psi(x+0)=$ $\alpha(x+0)$ and $\psi(x-0)=\alpha(x-0)$. By means of these relations, (12) can be reduced to the expressions given above for $\phi(x)$ and $\psi(x)$.

It is sometimes possible to express $T[f]$ in terms of the Young modification of the Stieltjes integral,* which is defined by

$$
\begin{aligned}
Y \int_{a}^{b} f d \alpha= & \lim \sum_{i=1}^{n}\left\{f\left(\xi_{i}\right)\left[\alpha\left(x_{i}-0\right)-\alpha\left(x_{i-1}+0\right)\right]\right. \\
& +f\left(x_{i-1}\right)\left[\alpha\left(x_{i-1}+0\right)-\alpha\left(x_{i-1}\right)\right] \\
& \left.+f\left(x_{i}\right)\left[\alpha\left(x_{i}\right)-\alpha\left(x_{i}-0\right)\right]\right\},\left(x_{i-1}<\xi_{i}<x_{i}\right) .
\end{aligned}
$$

Conditions for the equivalence of (5) and (13) are found as above by comparing the values of these expressions for the same specializations of the function $f(x)$. They are

* W. H. Young, Integration with respect to a function of bounded variation, Proceedings of the London Mathematical Society, (2), vol. 13 (1913), p. 113. As defined by Young, the integral is obtained by taking the limit as the maximum length of the subintervals approaches zero. The integral thus defined is of a weaker type than that given in (13). 


$$
\phi(x)=\psi(x+0)-\psi(x-0), \quad \psi(x)=\psi(x+0) .
$$

The conditions (14) place a greater restriction on $T$ than the corresponding conditions for expressing $T$ as a single Stieltjes integral of the Dushnik type or of the mean type. Consequently the Young-Stieltjes integral is not as well adapted to representing an operation $T[f]$ as these other modifications. The same remark applies to the Lebesgue-Stieltjes integral, which agrees with the $\mathrm{Y}$-integral in this case.

The fact that a linear functional operation $T[f]$ on the class of functions having discontinuities of the first kind is not always expressible as a Lebesgue-Stieltjes integral or as an ordinary Stieltjes integral of $f(x)$ with respect to a function of bounded variation may be shown in a different manner. Let $f_{n}(x)=1$ or 0 according as $0 \leqq x \leqq 1 / n$ or $1 / n<x \leqq 1$, and let $f_{0}(0)=1, f_{0}(x)=0$ elsewhere in $(0,1)$; and take as the operation $T$ the mean integral, $(m) \int_{0}^{1} f d \alpha$, of $f$ with respect to a function of bounded variation $\alpha(x)$ which is discontinuous on the right at $x=0$. Then $f_{1} \geqq f_{2} \geqq f_{3} \geqq \cdots \rightarrow f_{0}$, and

$$
\text { (m) } \quad \begin{aligned}
\int_{0}^{1} f_{0} d \alpha & =\frac{1}{2}[\alpha(0+0)-\alpha(0)], \\
(m) \quad \int_{0}^{1} f_{n} d \alpha & =\frac{1}{2}\left[\alpha\left(\frac{1}{n}\right)+\alpha\left(\frac{1}{n}+0\right)\right]-\alpha(0) \\
& \rightarrow \alpha(0+0)-\alpha(0),
\end{aligned}
$$

so that $(m) \int f_{n} d \alpha$ does not converge to $(m) \int f_{0} d \alpha$. But the LS-integral and the ordinary Stieltjes integrals possess the property that $\int f_{n} d \alpha$ converges to $\int f_{0} d \alpha$ if $f_{n} \rightarrow f_{0}$ monotonically, ${ }^{*}$ and therefore these integrals cannot be used to represent this operation $T$.

The University of Michigan

* See P. J. Daniell, Annals of Mathematics, (2), vol. 19 (1918), p. 280. 